\title{
A precessing molecular jet signaling an obscured, growing supermassive black hole in NGC 1377? ${ }^{\star, \star \star}$
}

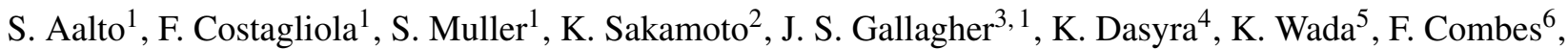 \\ S. García-Burillo ${ }^{7}$, L. E. Kristensen ${ }^{8}$, S. Martín ${ }^{9}, 10,11$, P. van der Werf ${ }^{12}$, A. S. Evans ${ }^{13}$, and J. Kotilainen ${ }^{14}$
}

(Affiliations can be found after the references)

Received 29 October 2015 / Accepted 23 February 2016

\begin{abstract}
With high resolution $\left(00^{\prime} 25 \times 0\right.$ ' 18$)$ ALMA CO 3-2 $(345 \mathrm{GHz})$ observations of the nearby $\left(D=21 \mathrm{Mpc}, 1^{\prime \prime}=102\right.$ pc $)$, extremely radio-quiet galaxy NGC 1377, we have discovered a high-velocity, very collimated nuclear outflow which we interpret as a molecular jet with a projected length of $\pm 150 \mathrm{pc}$. The launch region is unresolved and lies inside a radius $r<10 \mathrm{pc}$. Along the jet axis we find strong velocity reversals where the projected velocity swings from $-150 \mathrm{~km} \mathrm{~s}^{-1}$ to $+150 \mathrm{~km} \mathrm{~s}^{-1}$. A simple model of a molecular jet precessing around an axis close to the plane of the sky can reproduce the observations. The velocity of the outflowing gas is difficult to constrain due to the velocity reversals but we estimate it to be between 240 and $850 \mathrm{~km} \mathrm{~s}^{-1}$ and the jet to precess with a period $P=0.3-1.1 \mathrm{Myr}$. The CO emission is clumpy along the jet and the total molecular mass in the high-velocity $\left( \pm\left(60\right.\right.$ to $\left.\left.150 \mathrm{~km} \mathrm{~s}^{-1}\right)\right)$ gas lies between $2 \times 10^{6} M_{\odot}$ (light jet) and $2 \times 10^{7} M_{\odot}$ (massive jet). There is also CO emission extending along the minor axis of NGC 1377. It holds $>40 \%$ of the flux in NGC 1377 and may be a slower, wide-angle molecular outflow which is partially entrained by the molecular jet. We discuss the driving mechanism of the molecular jet and suggest that it is either powered by a (faint) radio jet or by an accretion disk-wind similar to those found towards protostars. It seems unlikely that a massive jet could have been driven out by the current level of nuclear activity which should then have undergone rapid quenching. The light jet would only have expelled $10 \%$ of the inner gas and may facilitate nuclear activity instead of suppressing it. The nucleus of NGC 1377 harbours intense embedded activity and we detect emission from vibrationally excited $\mathrm{HCN} J=4-3 v_{2}=1 f$ which is consistent with hot gas and dust. We find large columns of $\mathrm{H}_{2}$ in the centre of NGC 1377 which may be a sign of a high rate of recent gas infall. The dynamical age of the molecular jet is short ( $<1 \mathrm{Myr}$ ), which could imply that it is young and consistent with the notion that NGC 1377 is caught in a transient phase of its evolution. However, further studies are required to determine the age of the molecular jet, its mass and the role it is playing in the growth of the nucleus of NGC 1377.
\end{abstract}

Key words. galaxies: evolution - galaxies: active - galaxies: individual: NGC 1377 - galaxies: jets - galaxies: ISM - ISM: molecules

\section{Introduction}

The growth of central baryonic mass concentrations and their associated supermassive black holes (SMBHs) are key components of galaxy evolution (e.g. Kormendy \& Ho 2013). The underlying processes behind the evolution of the SMBH and how it is linked to its host galaxy and its interstellar gas are, however, not well understood. In addition, it is not clear how SMBHs can grow despite the energy/luminosity of accretion that leads to gas expulsion from the region. Massive molecular outflows powered by active galactic nuclei (AGNs) and bursts of star formation are suggested as being capable of driving out a large fraction of the galaxy's cold gas reservoir in only a few tens of Myr (e.g. Nakai et al. 1987; Walter et al. 2002; Feruglio et al. 2010, 2015; Sturm et al. 2011; Aalto et al. 2012a, 2015b; Combes et al. 2013; Bolatto et al. 2013; Cicone et al. 2014; Sakamoto et al. 2014; García-Burillo et al. 2014; Alatalo 2015). To maintain nuclear activity and growth, an inflow of gas from larger radii is therefore required.

^ Based on observations carried out with the ALMA Interferometer. ALMA is a partnership of ESO (representing its member states), NSF (USA) and NINS (Japan), together with NRC (Canada) and NSC and ASIAA (Taiwan), in cooperation with the Republic of Chile. The Joint ALMA Observatory is operated by ESO, AUI/NRAO and NAOJ.

$\star \star$ FITS files of the reduced data cubes are only available at the CDS via anonymous ftp to cdsarc.u-strasbg. fr (130.79.128.5) or via http://cdsarc.u-strasbg.fr/viz-bin/qcat?]/A+A/590/A73
Cold molecular gas has been proposed as an important source of fuel for SMBH growth since the accretion of hot gas is meant to be inefficient and slow (Blandford \& Begelman 1999; Nayakshin 2014). However, it is not known how the cold gas is deposited into the inner nucleus of the galaxy. This angular momentum problem is similar for the growth of SMBHs and the formation of stars (Larson 2010) and is even more severe for SMBHs because they are smaller than stars in relation to the size of the system in which they form. Thus, the mass that SMBHs may achieve is likely to be strongly regulated by the efficiency of angular momentum transfer during the fuel process. In protostars there is strong evidence of a physical link between infall and outflow (e.g. Arce et al. 2007) and angular momentum can be transferred by molecular jets and outflows. A link betwen infall and outflow seems to also exist for galaxy nuclei and AGNs (e.g. Davies et al. 2014; García-Burillo et al. 2014)

Chaotic inflows of cold gas clumps with randomly oriented angular momenta have been suggested as alternatives to large scale disks in feeding the growth of the SMBH (King \& Pringle 2007; Gaspari et al. 2013; Nayakshin et al. 2012). In this scenario, SMBH growth may occur primarily through multiple small-scale accretion events, rather than continous accretion (e.g. King \& Pringle 2007) leading to AGN luminosity variations on time scales of $10^{3}-10^{6} \mathrm{yr}$ (Hickox et al. 2014). A somewhat contrasting picture is that angular momentum may be effectively transported by, for example, bars and spiral density waves on large and small scales (see e.g. discussion in 
García-Burillo et al. 2014). AGN luminosity and nuclear growth is therefore expected to vary depending on the interplay between mode of accretion, outflow, and winds.

To test how gas inflow and the feedback of central activity influences the growth of SMBHs it is important to study galaxies in early or transient phases of their nuclear evolution. NGC 1377 is a likely example of such a system. It belongs to a small subset of galaxies that has a pronounced deviation from the well-known radio-to-far-infrared (FIR) correlation, having excess FIR emission compared to the radio $(q>3$; $q=\log \left[\mathrm{FIR} / 3.75 \times 10^{12} \mathrm{~Hz}\right] / S_{v}(1.4 \mathrm{GHz})$; Helou et al. 1985). These FIR-excess and radio-quiet galaxies are rare. Roussel et al. (2003) find that they represent a small fraction (1\%) of an infrared flux-limited sample in the local universe, such as the IRAS Faint Galaxy Sample. Their scarcity is likely an effect of the short time spent in the FIR-excess phase, making them ideal targets for studies of transient stages of AGN, starburst, and feedback.

\subsection{The extremely radio-quiet FIR-excess galaxy NGC 1377}

NGC 1377 is a member of the Eridanus galaxy group at an estimated distance of $21 \mathrm{Mpc}\left(1^{\prime \prime}=102 \mathrm{pc}\right)$ and has a FIR luminosity of $L_{\mathrm{FIR}}=4.3 \times 10^{9} L_{\odot}$ (Roussel et al. 2003). In stellar light, NGC 1377 has the appearance of a regular lenticular galaxy (de Vaucouleurs et al. 1991) although Heisler \& Vader (1994) and Roussel et al. (2006) find a faint dust lane that extends along the southern part of the minor axis.

NGC 1377 is the most radio-quiet, FIR-excess galaxy known to date with radio synchrotron emission being deficient by at least a factor of 37 with respect to normal galaxies (Roussel et al. 2003, 2006). Interestingly, H II regions are not detected through near-infrared hydrogen recombination lines or thermal radio continuum even though faint optical emission lines are present (Roussel et al. 2003, 2006). Deep mid-infrared silicate absorption features suggest that the nucleus is enshrouded by large masses of dust (e.g. Spoon et al. 2007). This supports the notion that NGC 1377 may be in a transient phase of its evolution since a more advanced nuclear activity is expected to have cleared out the enshrouding material. It has been suggested that the compact IR nucleus may be the site of a nascent $(t<1 \mathrm{Myr})$ opaque starburst (Roussel et al. 2003, 2006) or of a buried AGN (Imanishi 2006; Imanishi et al. 2009).

High resolution SMA CO 2-1 observations revealed a large central concentration of molecular gas and a massive molecular outflow (Aalto et al. 2012b) that appeared to be young ( $1.4 \mathrm{Myr})$. The extremely high nuclear dust and gas obscuration of NGC 1377 aggravates the determination of the nature of the nuclear activity and the driving force of the molecular outflow, but the extraordinary radio deficiency implies transient nuclear activity.

We used the Atacama Large Millimeter/submillimeter Array (ALMA) to observe CO 3-2 at high resolution in NGC 1377 aiming to determine the nature of the buried source and the structure and evolutionary status of the outflow. Here we present the discovery of a high-velocity, extremely collimated and precessing molecular jet in NGC 1377. Our results show that the nuclear source is likely an AGN and that we are either witnessing a faint radio jet driving a molecular collimated outflow, or a jet powered by cold accretion. The nuclear activity of NGC 1377 may be fading, or the large nuclear concentration of gas and dust signify that the major AGN event has not yet occured. We also discuss how the gas transfer in the moleular jet may instead foster gas recycling and how this process may promote $\mathrm{SMBH}$ growth.

\section{Observations}

Observations of the CO $J=3-2$ line were carried out with ALMA (with 35 antennas in the array) on 2014 August 12, for about half an hour on-source and with good atmospheric conditions (precipitable amount of water vapour of $\sim 0.5 \mathrm{~mm}$ ). The phase centre was set to $\alpha=03: 36: 39.074$ and $\delta=$ -20:54:07.055 (J2000).

The correlator was set up to cover two bands of $1.875 \mathrm{GHz}$ in spectral mode, one centred at a frequency of $\sim 344.0 \mathrm{GHz}$ to cover the $\mathrm{CO} J=3-2$ line (in the lower side band), and the other centred at $354.3 \mathrm{GHz}$ to cover the $\mathrm{HCO}^{+} J=4-3$ and $\mathrm{HCN}$ $J=4-3 v=0$ and $v=1 f$ lines (in the upper side band). The velocity resolution for these bands was $1.0 \mathrm{~km} \mathrm{~s}^{-1}$ after Hanning smoothing. In addition, two $2 \mathrm{GHz}$ bands were set up in continuum mode, i.e., with a coarser velocity resolution of $\sim 27 \mathrm{~km} \mathrm{~s}^{-1}$, centred at 342.2 and $356 \mathrm{GHz}$, respectively.

The bandpass of the individual antennas was derived from the quasar J0423-0120. The quasar J0340-2119 ( 0.3 Jy) was observed regularly for complex gain calibration. The absolute flux scale was calibrated using the quasar J0334-401. The flux density for J0334-401 was extracted from the ALMA fluxcalibrator database.

After calibration within the CASA reduction package, the visibility set was imported into the AIPS package for further imaging. The synthesized beam is $0.25 \times 00^{\prime} 18(25 \times 18 \mathrm{pc}$ for NGC 1377) with Briggs weighting (parameter robust set to 0.5 ) and the resulting data has a sensitivity of $0.8 \mathrm{mJy}$ per beam in a $10 \mathrm{~km} \mathrm{~s}^{-1}$ (12 MHz) channel width.

\section{Results}

\subsection{CO 3-2 moment maps}

The CO 3-2 integrated intensity (moment 0 ) map, velocity field (moment 1) and dispersion map (moment 2) are presented in Fig 1. We smoothed to two channel resolution, then for the moment 0 map we clipped at the $3 \sigma$ level, and for the moment 1 and 2 maps we clipped at $4 \sigma$. The velocity centroids were determined through a flux-weighted first moment of the spectrum of each pixel, therefore assigning one velocity to a spectral structure. The dispersion was determined through a flux-weighted second moment of the spectrum of each pixel. This corresponds to the one dimensional velocity dispersion (i.e. the FWHM line width of the spectrum divided by 2.35 for a Gaussian line profile)

The integrated intensity map shows centrally peaked emission with some structure extending radially from the centre up to a radius of $\sim 1^{\prime \prime} .5(150 \mathrm{pc})$. An estimated $11 \%$ of the emission is emerging from the inner $25 \times 18 \mathrm{pc}$ (see Table 1 ). The velocity field is complex and implies that the maximum velocity shifts occur outside the nucleus. There is evidence for a shallow eastwest velocity gradient around the nucleus. The moment 2 map reveals a striking, narrow $3^{\prime \prime}$ long feature of high dispersion.

The $\mathrm{CO}$ emission clearly delineates two separate structures (Fig. 2): an extremely well collimated jet-like structure, which, essentially, is visible at high velocities and large-scale emission at low velocities, which surrounds the high velocity jet-like feature. We interpret the high velocity feature as a molecular jet (see Sect. 4.1) and we will refer to it as such in the text below. 

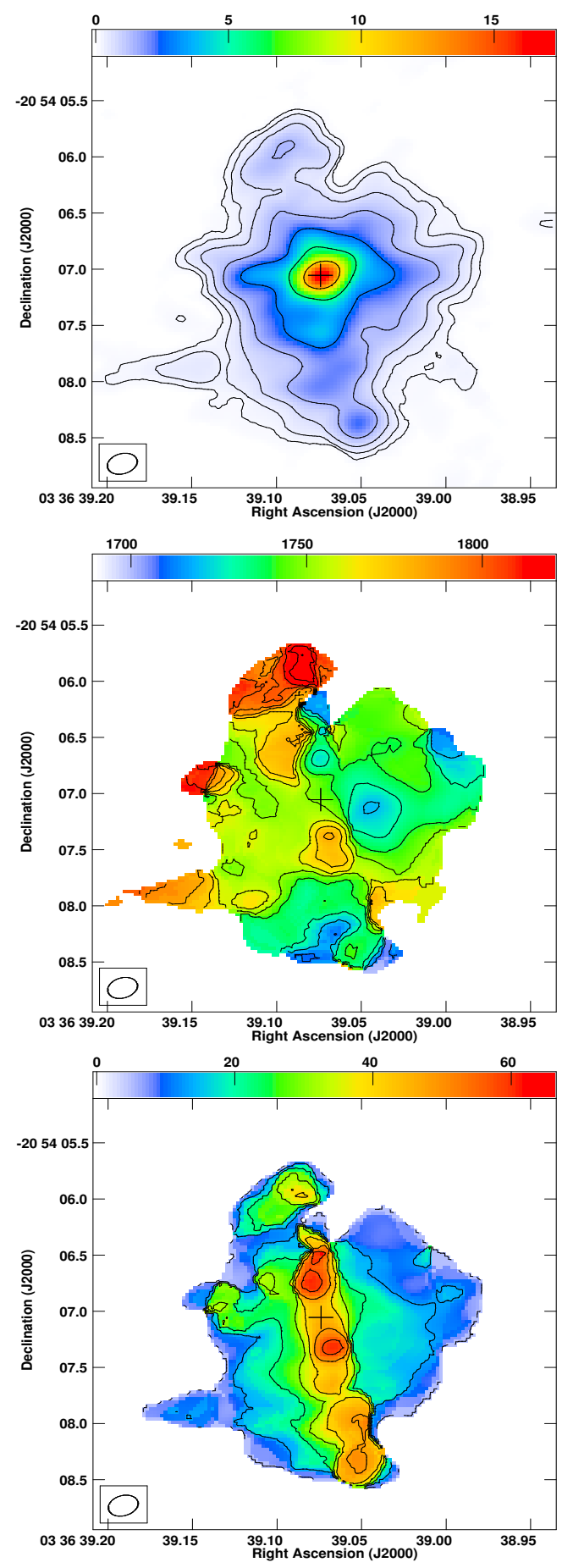

Fig. 1. CO 3-2 moment maps. Top: integrated intensity (mom0) where contours are $1.7 \times(1,2,4,8,16,32,64) \mathrm{Jy} \mathrm{km} \mathrm{s}^{-1}$ beam $^{-1}$ Colours range from 0 to $172 \mathrm{Jy} \mathrm{km} \mathrm{s}^{-1}$ beam $^{-1}$. Centre: velocity field (mom1) where contours range from $1690 \mathrm{~km} \mathrm{~s}^{-1}$ to $1820 \mathrm{~km} \mathrm{~s}^{-1}$ in steps of $10 \mathrm{~km} \mathrm{~s}^{-1}$. Bottom: dispersion map (mom2) where contours are $4.4 \times(1,3,5,7,9,11,13) \mathrm{km} \mathrm{s}^{-1}$. Colours range from 0 to $66 \mathrm{~km} \mathrm{~s}^{-1}$. The cross indicates the position of the $345 \mathrm{GHz}$ continuum peak (see Table 1).

\subsection{The high velocity gas - a molecular jet}

The high velocity (projected velocities $60-150 \mathrm{~km} \mathrm{~s}^{-1}$ ) gas (Fig. 2) is aligned in a $\pm 1^{\prime \prime} .5( \pm 150 \mathrm{pc})$ long, highly collimated, jet. It has an unresolved width $(d<20 \mathrm{pc}-$ set by the limit of our resolution) and a position angle PA $=10^{\circ}$. In Fig. 2 we show

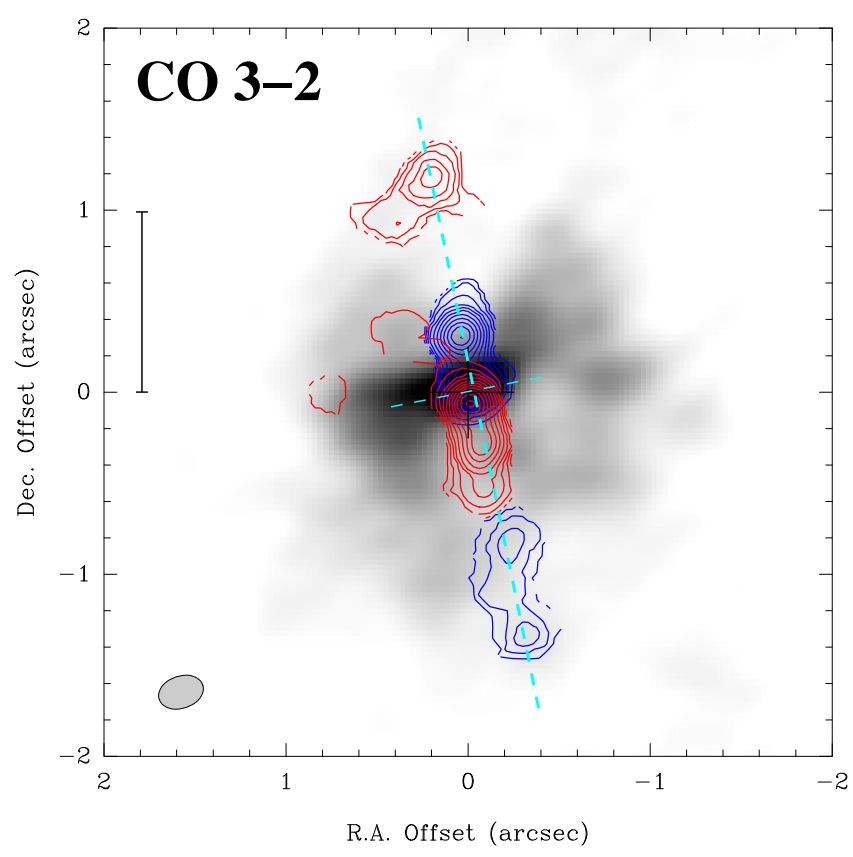

Fig. 2. CO 3-2 integrated intensity image where emission close to systemic velocity $\left(1700-1760 \mathrm{~km} \mathrm{~s}^{-1}\right.$ ) is shown in greyscale (ranging from 0 to $\left.70 \mathrm{Jy} \mathrm{km} \mathrm{s}^{-1}\right)$. The high velocity $\left( \pm 80\right.$ to $\left.\pm 150 \mathrm{~km} \mathrm{~s}^{-1}\right)$ emission from the molecular jet is shown in contours (with the red and blue showing the velocity reversals). The contour levels are $1.0 \times(1,2,3,4$, $5,6,7,8,9) \mathrm{Jy} \mathrm{km} \mathrm{s}^{-1}$ beam $^{-1}$. The dashed lines indicate the jet axis and the inferred orientation of the nuclear disk. The $\mathrm{CO} 3-2$ beam is shown as a grey ellipse in the bottom left corner. The vertical bar indicates a scale of $100 \mathrm{pc}$.

Table 1. CO 3-2 flux densities and molecular masses.

\begin{tabular}{|c|c|}
\hline Position $^{a}($ J2000) & $\begin{array}{l}\alpha: 03: 36: 39.073\left( \pm 00^{\prime} 01\right) \\
\delta:-20: 54: 07.05( \pm 0.01)\end{array}$ \\
\hline $\begin{array}{l}\text { Peak flux density } \\
\text { Flux }\end{array}$ & $156 \pm 1\left(\mathrm{mJy} \mathrm{beam}^{-1}\right)$ \\
\hline $\begin{array}{l}\text { (central beam) } \\
\text { (molecular jet) }^{c} \\
(\text { whole map) }\end{array}$ & $\begin{array}{l}17.4 \pm 0.05\left(\mathrm{Jy} \mathrm{km} \mathrm{s}^{-1} \text { beam }^{-1}\right) \\
23.2 \pm 0.5\left(\mathrm{Jy} \mathrm{km} \mathrm{s}^{-1}\right) \\
159 \pm 0.5\left(\mathrm{Jy} \mathrm{km} \mathrm{s}^{-1}\right)\end{array}$ \\
\hline $\begin{array}{l}\text { Molecular mass }^{d} \\
\text { (central beam) } \\
\text { (molecular jet) } \\
\text { (whole map) }\end{array}$ & $\begin{array}{l}1.8 \times 10^{7} M_{\odot} \\
2.3 \times 10^{7} M_{\odot} \\
16 \times 10^{7} M_{\odot}\end{array}$ \\
\hline
\end{tabular}

Notes. Listed errors are $1 \sigma$ rms. ${ }^{(a)}$ The position of the peak $345 \mathrm{GHz}$ continuum emission and of the $\mathrm{CO} 3-2$ integrated intensity. The peak $T_{\mathrm{B}}$ is at $\alpha: 03: 36: 39.072 \delta:-20: 54: 07.06$ at $V_{\mathrm{c}}=1730 \mathrm{~km} \mathrm{~s}^{-1}$. ${ }^{(b)}$ The Jy to $\mathrm{K}$ conversion in the $0^{\prime} .25 \times 00^{\prime} 18$ beam is $1 \mathrm{~K}=4.6 \mathrm{mJy}$. The peak $T_{\mathrm{B}}$ is $34 \mathrm{~K}$ corresponding to $156 \mathrm{mJy} .{ }^{(c)}$ The jet flux is integrated from $\pm(60$ to 200$) \mathrm{km} \mathrm{s}^{-1}$ where the blueshifted flux is 5.5 and the redshifted $17.7 \mathrm{Jy} \mathrm{km} \mathrm{s}^{-1}$. ${ }^{(d)}$ The $\mathrm{H}_{2}$ mass $M\left(\mathrm{H}_{2}\right)=1 \times 10^{4} S(\mathrm{CO} 1-0) \Delta v D^{2}(D$ is the distance in Mpc, $S \Delta v$ is the integrated CO 1-0 line flux in Jy km s${ }^{-1}$ ) for a conversion factor $N\left(\mathrm{H}_{2}\right) / I(\mathrm{CO} 1-0)=2.5 \times 10^{20} \mathrm{~cm}^{-2}$. Since we have $\mathrm{CO} 3-2$ we have to correct for the frequency dependence of the brightness temperature conversion. If $\mathrm{CO} 3-2$ and 1-0 have the same brightness temperature (thermal excitation, optically thick) the correction factor is $1 / 9$. However, usually the $\mathrm{CO}$ emission is subthermally excited and the brightness temperature ratio is expected to be about 0.5 for a giant molecular cloud. Hence the correction factor we apply is $1 / 4.5$ and $M\left(\mathrm{H}_{2}\right)=2.2 \times 10^{3} S(\mathrm{CO} 3-2) \Delta v D^{2}$. The inferred $\mathrm{H}_{2}$ column density in the central beam is $N\left(\mathrm{H}_{2}\right)=3 \times 10^{24} \mathrm{~cm}^{-2}$. 
that near the nucleus (within 0.5 ) emission at redshifted velocities is on the southern side and emission at blueshifted velocities are found to the north. Further along the axis (beyond 0.'5) this reverses.

\subsection{Systemic and low-velocity gas}

The systemic and low-velocity gas (projected velocities 0-60 $\mathrm{km} \mathrm{s}^{-1}$ ) consists of a bright central disk-like feature with $\mathrm{PA}=105^{\circ} \pm 5^{\circ}$ and larger scale emission extending primarily along the minor axis of NGC 1377. Along the PA of $105^{\circ} \pm 5^{\circ}$ there is an east-west velocity shift of $\sim 50 \mathrm{~km} \mathrm{~s}^{-1}$. The lowvelocity emission surrounds the molecular high-velocity jet in a butterfly-like pattern (Fig. 2). Most of the CO 3-2 flux of NGC 1377 emerges from this minor axis structure (Table 1). The minor axis extent of the systemic emission is similar to that of the high-velocity molecular jet, but we note that at zero velocities, negatives in the map indicate that some flux is missing from extended emission. The maximum recoverable scale of our observations is of the order of $\sim 5^{\prime \prime}$.

\subsection{Position-velocity diagrams}

In Fig. 3 we present six position-velocity (PV) diagrams to show the distinct structure of the high-velocity emission in relation to that of the systemic and low-velocity gas.

The PV diagram along the jet axis (A) shows the velocity reversals. Near the nucleus the highest velocity is blueshifted to the north and redshifted to the south. The maximum velocities occur about 0.25 (25 pc) away from the nucleus. Further away $(1$ '.2) the highest velocity is now redshifted on the north side and blueshifted to the south.

The PV diagram also shows that the $\mathrm{CO}$ emission peaks strongly in the nucleus and that the emission along the jet axis is clumpy. The clumps are unresolved in the CO 3-2 beam and from the Jy to $\mathrm{K}$ conversion in Table 1 we find that the clumps have brightness temperatures of $T_{\mathrm{B}}(\mathrm{CO} 3-2)=1-8 \mathrm{~K}$. The CO 3-2 line widths of the gas clumps in the jet are high ranging from 50 to $\sim 150 \mathrm{~km} \mathrm{~s}^{-1}$, which is evident in the PV diagram along the jet axis, as well as in the PV diagrams cut across the jet (C-F in Fig. 3).

We show four PV diagrams oriented perpendicular to the jet: two at distance \pm 0 '.25 from the nucleus (D and E) and two 1'.2 from the nucleus ( $\mathrm{C}$ and F). They were selected at the locations of highest velocities in the gas along the jet - and also to show the switch in orientation of the high-velocity gas (the velocity reversals). In PV diagrams D and $\mathrm{E}$ the distinction between the narrow, unresolved high-velocity gas from the extended emission (on scales of $\sim 2^{\prime \prime}(200 \mathrm{pc}$ )) of the low-velocity gas is clear. On the north side the high-velocity blueshifted emission in D is narrower than the redshifted high-velocity emission further out (C). A similar pattern is seen to the south where the narrow redshifted emission near the nucleus $(\mathrm{E})$ is more confined than the blueshifted emission further from the nucleus $(F)$. Here, there is also some emission at near-systemic velocities as well as an additional blueshifted component. Comparing D and $\mathrm{E}$ we find that the low-velocity gas to the north is slightly redshifted with respect to systemic velocity and to the south the emission is somewhat blueshifted.

We also present a PV diagram across the nucleus that is perpendicular to the jet component (B). Again, it shows the central concentration of the $\mathrm{CO} 3-2$ emission, broad unresolved emission on the nucleus, and narrower emission extending to the east
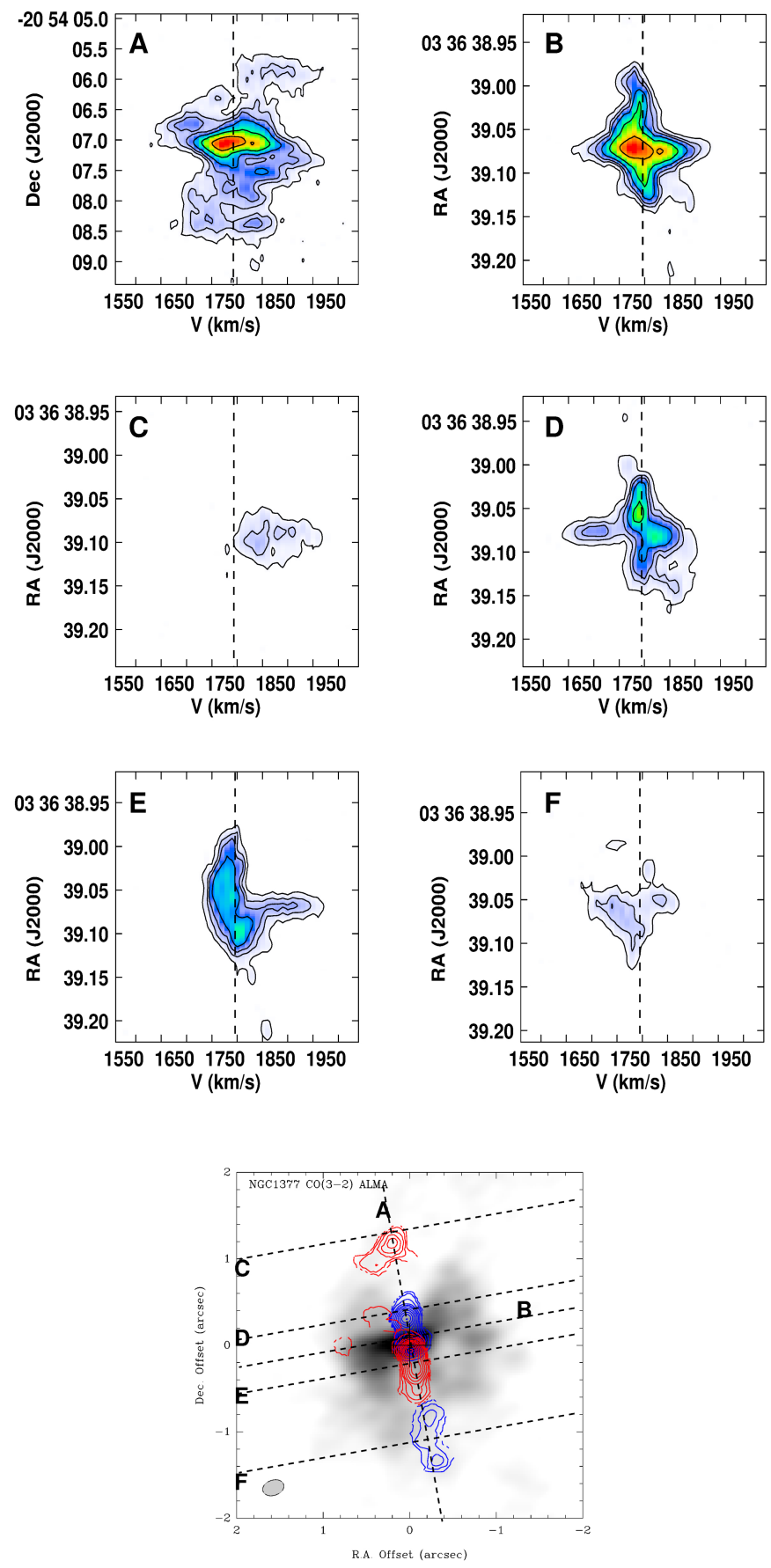

Fig. 3. Position-velocity (PV) diagrams showing gas velocities in six different slits: A) along the jet axis; B) perpendicular to the jet axis through the nucleus; C) perpendicular to the jet axis at 1 .' 2 to the north; D) perpendicular to the jet axis at 0.25 to the north; E) perpendicular to the jet axis at 0 ' 25 to the south; $\mathbf{F})$ perpendicular to the jet axis at $1 ., 2$ to the south. Contour levels are $3.1 \times(1,3,5,9,18,36)$ mJy beam $^{-1}$ thus the first level is at $4 \sigma$. The colour scale range from 2 to $156 \mathrm{mJy}^{-1}$ beam ${ }^{-1}$.

and west of the nucleus. There is a low velocity shift from east to west of $\pm 25 \mathrm{~km} \mathrm{~s}^{-1}$.

\subsection{Nuclear gas}

Velocities in the nucleus span a total of $300 \mathrm{~km} \mathrm{~s}^{-1}$. It is not clear what amount of this constitutes rotation of a circumnuclear disk and what amount stems from the outflowing gas in the jet. The velocities in the moment 1 map (Fig. 1) do not show 


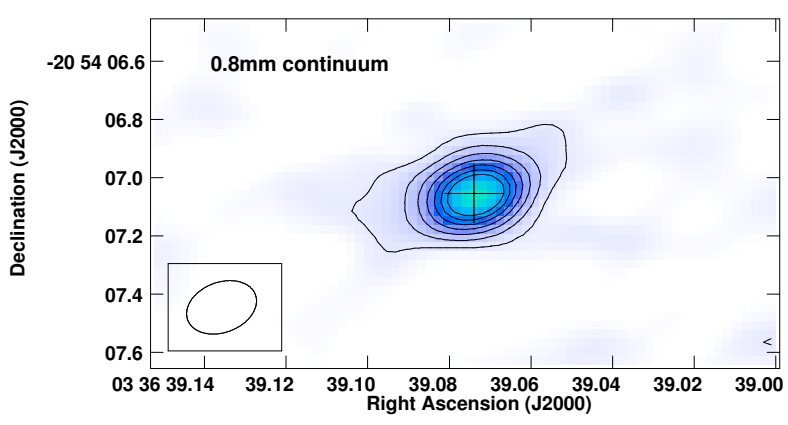

Fig. 4. $0.8 \mathrm{~mm}$ continuum (merged $342,349,356 \mathrm{GHz}$ line-free channels). Contour levels are $0.14 \times(1,2,3,4,5,6,7) \mathrm{mJy}_{\text {beam }}{ }^{-1}$. The lowest contour is at $3 \sigma$. The cross indicates the continuum peak position (see Table 1).

much rotation around the nucleus. The nuclear emission is broad but unresolved in space, and the velocity outside of the nucleus drops quickly with radius along the major axis of the galaxy, as is evident in PV diagram (B) in Fig. 3.

From the $\mathrm{CO}$ luminosity, we infer an $\mathrm{H}_{2}$ column density of $N\left(\mathrm{H}_{2}\right)=3 \times 10^{24} \mathrm{~cm}^{-2}$ (Table 1 ) towards the nucleus. This would imply that the nucleus of NGC 1377 is Compton thick and similar to the nuclei of other extremely obscured early type disk galaxies, such as NGC 4418 (Sakamoto et al. 2013; Costagliola et al. 2013), IC 860 and Zw049.057 (Falstad et al. 2015; Aalto et al. 2015a), but more studies are required to confirm this high $N\left(\mathrm{H}_{2}\right)$ for NGC 1377.

Apart from CO 3-2 we also detected $\mathrm{HCO}^{+}$and $\mathrm{H}^{13} \mathrm{CN} J=$ 4-3 and vibrationally excited HCN $J=4-3 v_{2}=1 f(T=$ $\left.E_{1} / k=1050 \mathrm{~K}\right)$ The vibrationally excited lines is a factor of 20-30 times fainter than $\mathrm{CO} 3-2$ in the nucleus, but its detection is consistent with a large $N\left(\mathrm{H}_{2}\right)$ and the presence of very hot gas and dust (Aalto et al. 2015a). We also detect lines at redshifted frequency $v=342.26$ and $344.5 \mathrm{GHz}$. The identification of these lines is not clear but we tentatively identify the first as $\mathrm{HC}^{15} \mathrm{~N}$ $J=4-3$ and the second either as vibrationally excited $\mathrm{HC}_{3} \mathrm{~N}$ $J=38-37 v_{4}=1, v_{7}=1$, or as $\mathrm{SO}_{2}$. We present spectra and a brief discussion of the line identification in Appendix A.

\subsection{Continuum}

We merged all line-free channels in our observations into a $0.8 \mathrm{~mm}$ continuum image (Fig. 4). It consists of a compact component and some extended emission. In the $0.25 \times 0 .{ }^{\prime} 18$ beam, the deconvolved FWHM size is $0.25 \times 0 . ' 09$ and a position angle PA $=104 \pm 5^{\circ}$. The continuum is faint $\left(1.3 \pm 0.1 \mathrm{mJy} \mathrm{beam}^{-1}\right.$ peak and $2.2 \pm 0.3 \mathrm{mJy}$ integrated). The $\mathrm{rms}$ is $0.045 \mathrm{mJy}$. The continuum and CO 3-2 peak in the same position, which we assume is the nucleus of the galaxy.

\section{Discussion}

\subsection{The high velocity gas: a precessing molecular jet?}

We interpret the high velocity CO 3-2 emission as emerging from a highly collimated and ordered molecular jet. The striking velocity reversals along its symmetry axis are consistent with those of jet precession (e.g. Rosen \& Smith 2004). The maximum velocity swings from 1590 to 1910 north of the nucleus (Fig. 3A) and from 1920 to 1650 to the south. Thus on average the shift is $300 \mathrm{~km} \mathrm{~s}^{-1}$. The velocity shifts to the north and south appear fairly symmetric, which suggests that the symmetry axis of the jet should be relatively close to the plane of the sky and thus launched from a highly inclined disk.

The $0.8 \mathrm{~mm}$ continuum image (Fig. 4) implies a nuclear disk of inclination $70^{\circ} \pm 10^{\circ}$ and a FWHM radius of $13 \mathrm{pc}$ (although we caution that the continuum emission is faint and only marginally resolved). In addition, the nuclear $\mathrm{CO}$ emission lines are broad with an unresolved dynamics, which is also consistent with the notion of a compact, highly inclined nuclear disk.

\subsubsection{Simple models}

The PV diagram along the symmetry axis of a precessing jet of constant outflow velocity shows the projected velocity oscillate ${ }^{1}$ as the jet alternates its direction towards and away from the observer. This is demonstrated schematically in Fig. 5 where we show the resulting PV diagram of a simple model with a precession angle $\theta=15^{\circ}$, the inclination of the precession axis is zero (i.e. in the plane of the sky), and the outflow velocity $v_{\text {out }}$ is constant. The precession has gone through slightly more than half a period. The first maximum velocity occurs 0.25 above the nucleus when the jet is most pointed towards us, implying that the jet close to the nucleus is seen at an angle.

We require higher spatial resolution to carry out proper model fits to the jet properties. However, for illustrative purposes we present two model maps in Fig. 6, showing what the model above would look like if we presented it in contour plot form i.e. similar to the high velocity contours in Fig. 2. We show two scenarios: one with precession angle $\theta=25^{\circ}$ and $v_{\text {out }}=260 \mathrm{~km} \mathrm{~s}^{-1}$, the other with $\theta=10^{\circ}$ and $v_{\text {out }}=600 \mathrm{~km} \mathrm{~s}^{-1}$, to demonstrate the effect of the precession angle on the high-velocity contour plots. Here the inclination of the precession axis to the declination axis is $10^{\circ}$ and, to the plane of the sky, it is zero. We assume a jet width of 0. . 4.

\subsubsection{Jet parameters}

The PV diagrams across the jet will show features that are broad in velocity if the cut includes the maximum projected velocity. For these velocities, the spatial extent will be the lowest and the emission will be narrowest near the base of the jet, while the emission at maximum velocity will be broader further away from the nucleus owing to the precession of the jet. This effect can be seen in Fig. 3 (panels C and D) where the blueshifted jet component near the nucleus in $\mathrm{D}$ is narrower than the redshifted jet component further away (to the north) from the centre. In addition, the position of the redshifted jet component is shifted to the east, compared to the blueshifted component. This also gives the jet precession direction implied in Fig. 3. The offset of the redshifted jet component to the north can be used to estimate the precession angle. The maximum velocity is expected to be completely aligned with the jet axis, but emission at lower redshifted velocities are coming in from the east (also showing the direction of the precession). The east-offset implies a precession angle $\theta=10^{\circ}-25^{\circ}$, but this is, of course, very uncertain since we only have slightly more than half a turn of the jet. The unprojected outflow velocity $v_{\text {out }}$ depends on $\theta$ and the observed maximum

\footnotetext{
1 We use the formalism by $\mathrm{Wu}$ et al. (2009) to describe the lineof-sight velocity change along the symmetry axis of the jet: $v_{\mathrm{LOS}}=$ $v_{\text {LSR }} \pm v_{\text {out }}\left[\cos \theta \sin i+\sin \theta \cos i \cos \left(2 \pi l / \lambda+\phi_{0}\right)\right]$, where $v_{\text {LOS }}$ is the observed line-of-sight velocity, $v_{\text {out }}$ is the outflow velocity in the jet, $i$ is the inclination of the jet symmetry axis to the plane of the sky, $\theta=$ precession angle, $l=$ distance from the nucleus, $\lambda=$ precession length scale, $\phi_{0}=$ initial phase at the nucleus.
} 

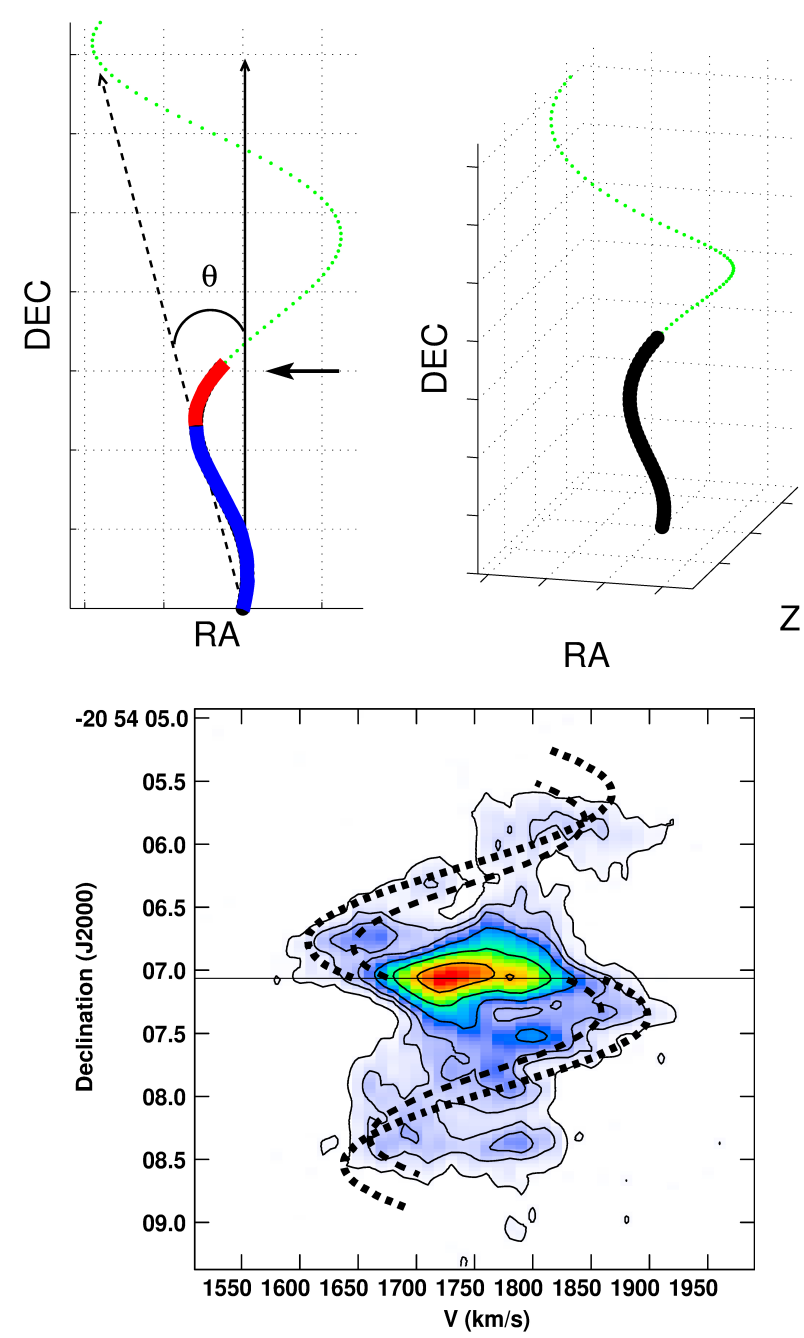

Fig. 5. Simple schematic jet model where we have rotated the jet symmetry axis from $\mathrm{PA}=10^{\circ}$ to $0^{\circ}$. Top: to the right, the northern part of the jet viewed face on. The curve indicates the pattern of the jet path on the sky and the blue and red colours indicate blue- and redshifted emission in the sight-line. The precession angle here is $\theta=15^{\circ}$ and the arrow indicates where the CO 3-2 emission in the jet ends. The right panel shows the jet viewed from an angle of $45^{\circ}$ to illustrate its $3 \mathrm{D}$ nature. Bottom: the observed PV diagram along the jet axis (panel A in Fig. 3 ) with the superposed tracks of a precessing jet of $\theta=15^{\circ}$ and outflowing velocity $v_{\text {out }}=390 \mathrm{~km} \mathrm{~s}^{-1}$ and $v_{\text {out }}=520 \mathrm{~km} \mathrm{~s}^{-1}$ indicated with dashed curves. (These values are within the range for $\theta$ and $v_{\text {out }}$ discussed in Sect. 4.1.2.) We assume $v_{\text {out }}$ to be constant and a jet without width.

projected velocity $v_{\text {proj }}$. This may either be done by selecting the velocity at the $3 \sigma$ contour or the velocity of the brighter clumps. This gives a rough span to $v_{\text {proj }}$ of $100-150 \mathrm{~km} \mathrm{~s}^{-1}$. The outflow velocity should, therefore, lie in the range $v_{\text {out }}=240-850 \mathrm{~km} \mathrm{~s}^{-1}$ with a precession period $P=0.3-1.1 \mathrm{Myr}$. The dynamical age of the full length of the molecular jet appears to be short. The jet can be traced out to $\sim 150 \mathrm{pc}$ and for $v_{\text {out }}=240-850 \mathrm{~km} \mathrm{~s}^{-1}$ the time scale ranges between $t=0.2$ and $0.7 \mathrm{Myr}$.

\subsubsection{Launch region}

The molecular jet emerges from the nucleus and its width is unresolved, which results in a launch region of the jet inside $r=10 \mathrm{pc}$. The nuclear rotation is also unresolved, but from the PV diagram we estimate a rotational velocity of $\sim 110 \mathrm{~km} \mathrm{~s}^{-1}$ and, if this occurs ar $r=10 \mathrm{pc}$, the rotational timescale is
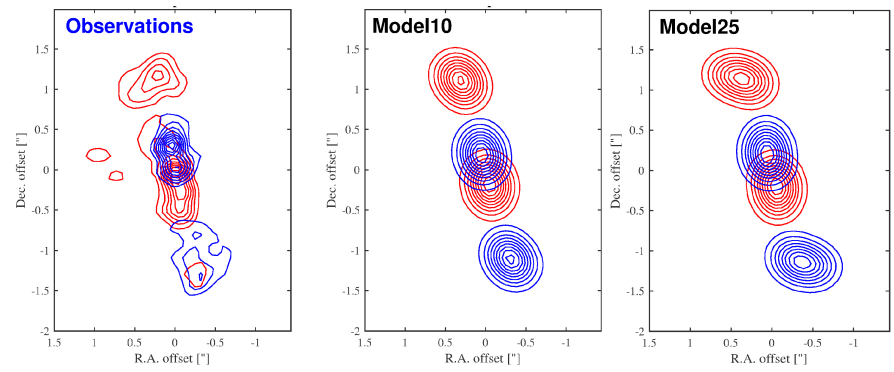

Fig. 6. Contour plots of integrated red- and blueshifted emission $\left(> \pm 60 \mathrm{~km} \mathrm{~s}^{-1}\right)$ for ALMA data (right) and model of precessing jet (left): the precession angle is $10^{\circ}$ (centre) and $25^{\circ}$ (right) the inclination of the model precession axis along the line of sight is close to zero.

$\sim 1$ Myr. The precession period must be longer than the rotational timescale of the jet-launching region and hence the jet is very likely launched close to the nucleus, within the inner few pc.

\subsubsection{Origin of precession}

Jet precession may occur in a variety of astrophysical objects, including low-mass star formation in the Galaxy (L1157 Gueth et al. 1996; Kwon et al. 2015; NGC 1333-IRAS4A Santangelo et al. 2015; L 1551 IRS 5 Fridlund \& Liseau 1994; IRAS 16293 2422 Kristensen et al. 2013); Galactic micro-quasars (SS433 Blundell \& Bowler 2005 and 1E 1740.7-2942 Luque-Escamilla et al. 2015), and AGN radio jets (e.g. Veilleux et al. 1993; Steffen 1997; Martí-Vidal et al. 2011; Pyrzas et al. 2015).

Jet precession may be caused by a warped accretion disk (e.g. Greenhill et al. 2003) i.e. by the misalignment between the spin orientation of the black hole and the surrounding accretion disk (e.g. Bardeen \& Petterson 1975; Lu \& Zhou 2005) and an accretion flow that is transporting in gas of misaligned angular momentum (Krolik \& Hawley 2015), but see also the discussion in Nixon \& King (2013). Alternatively, in a SMBH binary system, jet precession may be caused by geodetic precession of the spin axis of the primary rotating SMBH being misaligned with the binary total angular momentum, or by inner disk precession (owing to the tidal interaction of an inclined secondary SMBH). Interestingly, the presence of a nuclear gas and dust concentration and a precessing molecular jet can aid the coalescence of the SMBHs into resolving the "final-parsec problem" (Milosavljević $\&$ Merritt 2003; Aly et al. 2015). The post-starburst spectrum of NGC 1377 (Gallagher et al., in prep.) could perhaps be linked to a past merger event that left left an SMBH binary in the heart of NGC 1377.

\subsubsection{Other explanations}

In Appendix B we discuss potential alternative explanations for the high-velocity gas emission structure and why we find them less likely (with current information) than the precessing jet model presented here.

\subsection{Low-velocity gas}

The extremely simple jet model cannot explain all the features we see in the PV diagrams (Fig. 3). Perpendicular to the jet axis (panels $\mathrm{C}-\mathrm{F}$ in Fig. 3), we see the jet emission as a broad velocity feature and narrow in space. However, there is also more spatially extended emission at low velocities (panels D and E). In PV diagram A, the lower velocity emission occurs as straight 
lines to the north (in particular) but also in the south. There is also an extra component at $1800 \mathrm{~km} \mathrm{~s}^{-1}$ to the south next to the blueshifted part of the jet. This emission cannot be directly explained by a simple model of a precessing jet and may emerge from a background disk, a molecular wide-angle wind, or it is caused by interaction and entrainment by the jet.

For example a bow shock can arise by the formation of an internal working surface within the jet at positions of strong velocity discontinuity, and as the high velocity jet interacts with the surrounding medium (Raga \& Cabrit 1993; Gueth \& Guilloteau 1999; Cliffe et al. 1996; Santiago-García et al. 2009). The structure and velocity of the ambient gas may become complex owing to, for example, the action of the global bow shock and gas sweeping into the wakes of the jet turns. Dynamical simulations of precessing gaseous jets have been carried out by Raga et al. (2001). They present PV diagrams perpendicular to their simulated jet (their Fig. 5) and find that the transverse spatial extent of the emitting region is larger at lower radial velocities. In their simulations, this is due to the presence of bow shock wings trailing behind each internal working surface. These bow shocks result in transverse extended emission of low radial velocities which forms a "halo" component. There is a striking similarity between the Fig. 5 of Raga et al. (2001) and our PV diagrams that are perpendicular to the jet.

The low-velocity gas has redshifted velocities north-east of the jet and blueshifted velocities to the south-west. The angle between the most red- and blueshifted gas is PA $=40-45^{\circ}$ and the velocity shift is $60 \pm 20 \mathrm{~km} \mathrm{~s}^{-1}$. Apart from this gradient, there is no significant net shift in velocity between the north and the south (with deviations at the ends of the jet and at the edges of the map). There is a small (10-20 $\mathrm{km} \mathrm{s}^{-1}$ ) east-west gradient which is somewhat larger $\left(50 \mathrm{~km} \mathrm{~s}^{-1}\right)$ at the disk major axis.

The PA $=40-45^{\circ}$ velocity structure can be caused by the jet entraining and accelerating a very slow, wide-angle minor axis molecular outflow and/or that it is interacting with gas already entrained before. Another possibility is that there is a wind, which is unrelated to the jet and which originates in a disk warped about $20^{\circ}$, compared to the nuclear disk. This orientation is however not consistent with that of the optical dust absorption features south of the nucleus of NGC 1377 (Fig. 1 in Roussel et al. 2006; Fig. 4c in Heisler \& Vader 1994). The dust structures have a v-shaped morphology (opening angle of $\sim 90^{\circ}$ ) and are oriented almost perpendicular to the stellar disk. They may be caused, for example, by the precessing jet bow shocks.

\subsection{Comparing previous results for NGC 1377}

In our previous paper on NGC 1377(Aalto et al. 2012b), we suggested that the molecular outflow seen in $\mathrm{CO} 2-1$ is biconic with an opening angle of $60^{\circ}-70^{\circ}$, an outflow mass $>1 \times 10^{7} M_{\odot}$, and an outflow velocity of $140 \mathrm{~km} \mathrm{~s}^{-1}$. These observations were carried out with three times poorer spatial resolution and about ten times lower flux sensitivity than the ALMA CO 3-2 data presented here. The CO 2-1 dispersion map has a cross-like structure that we used as a basis to suggest the biconic outflow. In the ALMA data, high dispersion is found only along a structure that we now identify as a molecular jet.

It is interesting to note that the position angle of the outflow in the lower resolution $\mathrm{CO} 2-1$ map is around $\mathrm{PA}=40^{\circ}$, while the $\mathrm{CO} 3-2$ jet has a PA of $10^{\circ}$. The lower resolution SMA data has likely picked up the velocity shift in the low-velocity gas discussed above (Sect. 4.2) which we propose is caused by jet entrainment (or, less likely, an inclined wide-angle flow). Further studies will reveal more on the origin of this gas component.

\subsection{Mass and outflow rate}

The molecular jet: the molecular mass in the high velocity gas is estimated as $M_{\mathrm{j}}\left(\mathrm{H}_{2}\right)=2.3 \times 10^{7} M_{\odot}$, assuming a standard $\mathrm{CO}$ to $\mathrm{H}_{2}$ conversion factor (see Table 1). For $v_{\text {out }}$ between 240 and $850 \mathrm{~km} \mathrm{~s}^{-1}$, we estimate the mass outflow rate in the jet at 9-40 $M_{\odot} \mathrm{yr}^{-1}$. This results in a momentum flux of (14-200)L/c, which is very high and exceeds values typically seen in cases of AGN feedback (Cicone et al. 2014; García-Burillo et al. 2014). However, since we use a standard conversion factor, the $\mathrm{H}_{2}$ mass may have been overestimated. If the gas is turbulent, and the individual gas clouds unbound, the conversion factor may have to be adjusted down by a factor of 10 (e.g. Dahmen et al. 1998).

The low-velocity outflow: the mass and velocity in the lowvelocity outflow (Sect. 4.2) is difficult to estimate since there is the possibility of contamination by a background disk and the morphology and velocity structure are complicated. But if we assume that all the CO 3-2 flux above and below the stellar disk belong to the slow outflow, it would constitute $40 \%$ of the total CO 3-2 flux detected in NGC 1377. For a standard conversion factor (Table 1) this implies $M_{\text {slow }}\left(\mathrm{H}_{2}\right)=6 \times 10^{7} M_{\odot}$. About a third of this is associated with the entrained (alt inclined wind) part of the flow, with projected velocities $\pm 30 \mathrm{~km} \mathrm{~s}^{-1}$. A generous estimate of $v_{\text {out }} \sim 50 \mathrm{~km} \mathrm{~s}^{-1}$ over $100 \mathrm{pc}$ implies that $10 M_{\odot} \mathrm{yr}^{-1}$ may be lifted off the midplane of NGC 1377. This number is highly uncertain.

\subsection{What is powering the molecular jet?}

\subsubsection{Accretion}

Jets are generally identified with accretion (Blandford 1998; Konigl \& Pudritz 2000; Hujeirat et al. 2003; Sbarrato et al. 2014) and are likely launched by magnetohydrodynamic processes from the accretion disk and/or the central object. The molecular mass is a crucial ingredient in determining the energetics, nature, and evolutionary stage of the molecular jet. We have to resort to a $\mathrm{CO}$ to $M\left(\mathrm{H}_{2}\right)$ conversion factor to determine the molecular mass and we have two limiting cases: A massive jet where $M_{\mathrm{j}}\left(\mathrm{H}_{2}\right) \sim 10^{7} M_{\odot}$ or a light jet with $M_{\mathrm{j}}\left(\mathrm{H}_{2}\right) \sim 10^{6} M_{\odot}$. Below we discuss possible driving scenarios in relation to a massive or a light jet.

Entrainment by a radio jet. Powerful radio jets are launched when an SMBH is growing through hot accretion which is an inefficient accretion at low rates ( $<1 \%$ Eddington) (McAlpine et al. 2015). This is also referred to as radio mode AGN feedback. Radio jet production has been found for high Eddington rates where the jet powers do not exceed the bolometric luminosity of their AGNs (Sikora et al. 2013). The jet may entrain molecular gas from the disk of the host galaxy (NGC 1266 Alatalo et al. 2011; IC 5063 Morganti et al. 2015; M51 Matsushita et al. 2007 and NGC 1068 García-Burillo et al. 2014) or the molecular gas may form in the jet itself through rapid post-shock cooling (Morganti et al. 2015). Observed molecular gas distributions associated with these jets tend to be patchier than the more coherent molecular structure of NGC 1377. A relativistic radio jet ploughing through a thick disk of gas, is likely to heat and ionise it, and thus form a wide cocoon of multi-phase and turbulent gas mixture, as simulated by Wagner \& Bicknell (2011). As shown in Dasyra et al. (2015), this kind of cocoon is both pushing on the surrounding gas and has forward and scattered flows that may lead to complicated velocity patterns. 
However, NGC 1377 is the most radio-quiet (with respect to the IR luminosity) galaxy found so far and its radio power is very low. (A similar case with faint radio emission associated with molecular jets may be the double, collimated bipolar outflows of the luminous merger NGC 3256 Sakamoto et al. 2014). We can use the limit to the $1.4 \mathrm{GHz}$ radio luminosity (Roussel et al. 2006) and the relation between jet power and $1.4 \mathrm{GHz}$ luminosity (Bîrzan et al. 2008) to estimate the energy in a potential radio jet in NGC 1377 . We find that it amounts to $<10 \%$ of the mechanical energy in the massive molecular jet. A short burst of hot accretion in the nucleus may have led to the formation of a radio jet that then faded very rapidly without reacceleration of electrons in the jet itself. If the synchrotron life time is $t_{\mathrm{s}}=8 \times 10^{8} B^{-2} \gamma^{-1}$ (where $B=B$-field, $\gamma=$ Lorentz factor, Xu et al. 2000) a reasonable combination of $B$ and $\gamma$ can result in a jet lifetime of $0.5-1 \mathrm{Myr}$. Also, it is conceivable that heavily mass-loading a radio jet with dense molecular gas may lead to the quenching of the non-thermal radio emission. In addition, Godfrey \& Shabala (2016) recently suggested that jet power and radio luminosity may only be weakly correlated for cases where the jet energy is being used to, for example, drive shocks.

In the case of the light jet it is feasible that there would be enough radio power to carry the gas out without invoking a fading or underluminous radio jet.

Cold gas accretion. The jet may be a hydromagnetic disk-wind (or an accretion X-wind) similar to the extremely collimated molecular outflows found in accreting low-mass protostars (e.g. Konigl \& Pudritz 2000; Codella et al. 2014; Kristensen 2015). Its torque could efficiently extract disk angular momentum and gravitational potential energy from the molecular gas. The jet may be powered by accretion onto the central object and/or infalling gas onto the nuclear disk.

Assuming that the $5 \times 10^{9} L_{\odot}$ of NGC 1377 emerges from a growing SMBH, the accretion rate would be $\sim 10^{-3}-10^{-2} M_{\odot}$ $\mathrm{yr}^{-1}\left(L=\frac{\epsilon}{c^{2}} \mathrm{~d} M\right.$ where $\epsilon=0.1$ onto a $\left.10^{6} M_{\odot} \mathrm{SMBH}\right)$. This is $10 \%$ of the inferred Eddington luminosity of the SMBH (Aalto et al. 2012b) and is a relatively high rate, placing it in the quasar mode of accretion (McAlpine et al. 2015). But it may require an Eddington or super-Eddington accretion rate to produce the mass-outflow rate we see (even in the case of the light jet), implying that the level of SMBH accretion has dropped recently.

A jet may also be powered by accretion onto a nuclear disk. The wind energy is derived from the gravitational energy released from the disk through gas rotation and a coupled magnetic field. The extracted angular momentum allows cold molecular gas to sink further towards the nucleus. In the case of the massive jet, it is not clear how the current rotational energy of the disk could continue to sustain the outflow since $M_{\mathrm{j}}$ would be equal to that in the disk inside its launching region. The binding energy of the jet is similar to the binding energy of the disk and the outflow speed is at least twice that of the rotational velocity (unless the jet is actually launched very close to the nucleus from a Keplerian disk). In the case of the light jet, however, the jet-binding energy would be much less than that of the disk, and there would be enough rotational energy to sustain the outflow.

We note that the molecular jet is observed as being lumpy which may be due to internal and external shocks, or the condensations are gas clumps that originate in separate accretion/outflow events. If so, the energetics of the outflow may be different to that of the steady flow scenario we assume above.

\subsubsection{Other scenarios}

Radiation pressure from dust? Recent work by Ishibashi \& Fabian (2015) suggests that large momentum flux outflows $(>10 L / c)$ can be obtained in radiation pressure driven outflows if radiation trapping is taken into account. However, it is not clear how radiation pressure would result in a jet-like feature since it should give rise to a more wide-angle wind. Wada (2015) finds that dusty, biconical outflows (opening angles $45^{\circ}-60^{\circ}$ ) can be formed as a result of the radiation feedback from AGNs. It is conceivable that this may be happening in NGC 1377, in addition to the jet.

Starburst winds? In Aalto et al. (2012b) we discuss the faintness of the star formation tracers (such as optical, NIR and radio emission) of NGC 1377. We find that the upper limits on, for example, the $1.4 \mathrm{GHz}$ continuum imply that star formation falls short by at least one order of magnitude in explaining the momentum flux in the molecular outflow detected with the SMA.

\subsection{Is the molecular jet signaling nuclear growth or quenching?}

There is large molecular mass in the nucleus of NGC 1377, which appears to be linked to a current SMBH accretion at a respectable rate of $\sim 10 \%$ Eddington. So the question is: has the molecular jet action quenched the nuclear activity, or did it promote it?

Light jet: both scenarios discussed in Sect. 4.5.1 could power the jet and enable SMBH accretion. A light jet has removed only $10 \%$ of the disk mass while it may have transported a substantial amount of angular momentum away from the gas in the disk, allowing it to sink closer to the SMBH. The molecular jet offers a way for the cold gas to shed itself of excess angular momentum, which could promote nuclear accretion from a disk. In this scenario, the inflowing gas clouds do not have to have randomly oriented angular momenta to facilitate accretion. There is no evidence that star formation has hindered the gas flow toward the nucleus of NGC 1377. Instead there appears to be a mechanism that prevents stars from forming in the high gas surface density nuclear region. Higher resolution studies will hopefully find and resolve the inflowing gas component in NGC 1377.

Massive jet: current rates of accretion would be difficult to reconcile with a large mass outflow rate. Nuclear activity in the form of radio luminosity, or other forms of accretion luminosities, are low and are, perhaps, a signature of quenching. The turning off of the nuclear activity would have to have been abrupt since the molecular jet can be traced almost all the way down to the centre. Furthermore, the large masses of molecular gas are surprising since it is not clear why the activity would turn off with $30 \%$ of the nuclear fuel still in place. A possible explanation could be that there has been a recent substantial inflow of molecular gas.

The discussion above rests on the assumption that most of the FIR emission originates near the SMBH and is the result of the accretion. However, if the FIR emission is, instead, related to the jet-ISM interaction in an extremely dense medium, then the SMBH would be in the hot accretion mode instead, but with its synchrotron quenched by the interaction. If so, we are witnessing the early stages of jet feedback before it has cleared its environment. 


\subsubsection{What is the fate of the molecular gas?}

A precessing jet has the potential to impact and stir up a large volume of ambient gas. In NGC 1377 the jet appears to entrain gas in a slow moving outflow, possibly in combination with a wide-angle wind. It is, however, unlikely that the gas in the lowvelocity outflow can leave NGC 1377 since even an optimistic estimate of its outflow speed is below the bulge escape velocity $v_{\text {esc }}$ for NGC 1377 (Aalto et al. 2012b). Instead, gas may circulate back to the midplane of NGC 1377 where it could eventually participate in star formation or another cycle of nuclear growth.

The molecular jet appears to be a young structure with a dynamical age $<1$ Myr (Sect. 4.1.2). The estimated $v_{\text {out }}=$ $240-850 \mathrm{~km} \mathrm{~s}^{-1}$ is higher than $v_{\text {esc }}$ for NGC 1377 . We find no high-velocity molecular gas outside $200 \mathrm{pc}$ and this would be consistent with the notion that the jet has been caused by a recent accretion event in the nucleus. This would also be consistent with the high nuclear concentration of molecular gas. However, if the molecular gas becomes dissociated at $200 \mathrm{pc}$, we may simply be observing the inner denser part of an older outflow event and, if the gas is not slowing down, it may escape the galaxy. Yet another alternative is that the jet is rapidly decelerating and its gas is grinding to a halt at its end. The v-shaped optical dust lane is roughly 2-3 times longer than the molecular jet/outflow structure, which implies that the molecular jet is part of a somewhat older structure.

Our results demonstrate that outflows/jets even from lowpower AGNs can have substantial impact on the evolution of the galaxy, also beyond the innermost pc. We require the high resolution, dynamic range and sensitivity of ALMA to reveal the presence of the molecular jet and to separate it from surrounding emission. Determining the molecular mass in the jet will provide an important clue as to whether the jet is a signature of growth or quenching of the nuclear activity. More detailed studies will also reveal how the jet impacts its environment and entrains gas and dust.

\section{Conclusions}

With high resolution $(0.2 \times 0 . ' 18)$ ALMA CO 3-2 observations of the nearby extremely radio-quiet galaxy NGC 1377 , we have discovered a high velocity, collimated molecular jet with a projected length of $\pm 150 \mathrm{pc}$. Along the jet axis we find strong velocity reversals where the projected velocity swings from $-150 \mathrm{~km} \mathrm{~s}^{-1}$ to $+150 \mathrm{~km} \mathrm{~s}^{-1}$. A simple model of a molecular jet precessing around an axis close to the plane of the sky can reproduce the observations. The velocity of the outflowing gas is difficult to constrain due to the velocity reversals but we estimate it to be between 240 and $850 \mathrm{~km} \mathrm{~s}^{-1}$ and the jet to precess with a period $P=0.3-1.1 \mathrm{Myr}$.

The jet is launched close to the nucleus inside a radius $r<10 \mathrm{pc}$ and its molecular mass lies between $2 \times 10^{6}$ (light jet) and $2 \times 10^{7} M_{\odot}$ (massive jet) depending on which $\mathrm{CO}$ to $M\left(\mathrm{H}_{2}\right)$ conversion factor is adopted. There is also a wide-angle structure of $\mathrm{CO}$ emission along the minor axis which may be a slower molecular outflow. A substantial fraction of the $\mathrm{CO}$ flux is located here and the estimated mass of the minor axis outflow is $6 \times 10^{7} M_{\odot}$. Its velocity structure is consistent with parts of the wind being entrained by the jet, or that there is a molecular wind inclined by $30^{\circ}$ with respect to the jet.

We discuss potential powering mechanisms for the molecular jet. It may be gas entrained by a very faint radio jet, or it is driven by an accretion disk-wind similar to those found in protostars. It is important to better constrain the jet molecular mass.
Given the possibility of either a light or a heavy jet, it is difficult to draw conclusions on whether the jet is quenching the nuclear activity or, instead, is enabling it. The nucleus of NGC 1377 harbours intense embedded activity and, if the current IR luminosity is powered by a growing $\mathrm{SMBH}$, it would have an accretion rate of $\sim 10 \%$ Eddington. But the origin of the FIR luminosity still needs to be determined. The light jet would only have driven out $10 \%$ of the nuclear gas which should not (yet) significantly impact the fueling of the activity. It seems, however, unlikely that a massive jet could have been powered by the current activity and this may be a sign of rapid quenching. In this case, the large mass of $\mathrm{H}_{2}$ in the nucleus is surprising and may be caused by a recent massive influx of gas. A fraction of the outflowing gas may return to the inner region of NGC 1377 to fuel further nuclear growth.

NGC 1377 is the first galaxy with evidence for a precessing, highly collimated molecular jet. The extreme $q$-value for NGC 1377, the short apparent time-scale of the molecular jet $(<1 \mathrm{Myr})$, and the gas-rich nucleus are all signs consistent with the notion that we are seeing NGC 1377 in a transient phase of its evolution.

NGC 1377 offers a unique opportunity for detailed studies of the processes that feed, promote and quench nuclear activity in galaxies. Further studies are required to determine the age of the molecular jet, driving mechanism, its mass and the role it plays in the growth of the nucleus of NGC 1377.

Acknowledgements. This paper makes use of the following ALMA data: ADS/JAO.ALMA\#2012.1.00900.S. ALMA is a partnership of ESO (representing its Member States), NSF (USA) and NINS (Japan), together with NRC (Canada) and NSC and ASIAA (Taiwan), in cooperation with the Republic of Chile. The Joint ALMA Observatory is operated by ESO, AUI/NRAO and NAOJ. We thank the Nordic ALMA ARC node for excellent support. S.A. acknowledges support from the Swedish National Science Council grant 6212011-4143. F.C. acknowledges support from Swedish National Science Council grant 637-2013-7261 KS was supported by grant MOST 102-2119-M-001-011MY3 SGB thanks support from Spanish grant AYA2012-32295. JSG thanks the Chalmers University for the appointment of Jubileumsprofessor for 2015. S.A. thanks S. König, A. Hjalmarson, R. Haas and S. Bourke for discussions of the manuscript.

\section{References}

Aalto, S., Garcia-Burillo, S., Muller, S., et al. 2012a, A\&A, 537, A44 Aalto, S., Muller, S., Sakamoto, K., et al. 2012b, A\&A, 546, A68

Aalto, S., Costagliola, S. M. F., Gonzalez-Alfonso, E., et al. 2015a, A\&A, 584, A42

Aalto, S., Garcia-Burillo, S., Muller, S., et al. 2015b, A\&A, 574, A85

Alatalo, K. 2015, ApJ, 801, L17

Alatalo, K., Blitz, L., Young, L. M., et al. 2011, ApJ, 735, 88

Aly, H., Dehnen, W., Nixon, C., \& King, A. 2015, MNRAS, 449, 65

Arce, H. G., Shepherd, D., Gueth, F., et al. 2007, Protostars and Planets V, 245 Bardeen, J. M., \& Petterson, J. A. 1975, ApJ, 195, L65

Bîrzan, L., McNamara, B. R., Nulsen, P. E. J., Carilli, C. L., \& Wise, M. W. 2008, ApJ, 686, 859

Blandford, R. D. 1998, in AIP Conf. Ser. 431, eds. S. S. Holt, \& T. R. Kallman, 43

Blandford, R. D., \& Begelman, M. C. 1999, MNRAS, 303, L1

Blundell, K. M., \& Bowler, M. G. 2005, ApJ, 622, L129

Bolatto, A. D., Warren, S. R., Leroy, A. K., et al. 2013, Nature, 499, 450

Cabrit, S., \& Bertout, C. 1986, ApJ, 307, 313

Cicone, C., Maiolino, R., Sturm, E., et al. 2014, A\&A, 562, A21

Cliffe, J. A., Frank, A., \& Jones, T. W. 1996, MNRAS, 282, 1114

Codella, C., Cabrit, S., Gueth, F., et al. 2014, A\&A, 568, L5

Combes, F., García-Burillo, S., Casasola, V., et al. 2013, A\&A, 558, A124

Costagliola, F., Aalto, S., Sakamoto, K., et al. 2013, A\&A, 556, A66

Dahmen, G., Huttemeister, S., Wilson, T. L., \& Mauersberger, R. 1998, A\&A, 331,959

Das, V., Crenshaw, D. M., Hutchings, J. B., et al. 2005, AJ, 130, 945

Dasyra, K. M., Bostrom, A. C., Combes, F., \& Vlahakis, N. 2015, ApJ, 815, 34 
Davies, R. I., Maciejewski, W., Hicks, E. K. S., et al. 2014, ApJ, 792, 101 de Vaucouleurs, G., de Vaucouleurs, A., Corwin, Jr., H. G., et al. 1991, Third Reference Catalogue of Bright Galaxies (New York: Springer)

Falstad, N., González-Alfonso, E., Aalto, S., et al. 2015, A\&A, 580, A52

Feruglio, C., Maiolino, R., Piconcelli, E., et al. 2010, A\&A, 518, L155

Feruglio, C., Fiore, F., Carniani, S., et al. 2015, A\&A, 583, A99

Fridlund, C. V. M., \& Liseau, R. 1994, A\&A, 292, 631

García-Burillo, S., Combes, F., Usero, A., et al. 2014, A\&A, 567, A125

Gaspari, M., Ruszkowski, M., \& Oh, S. P. 2013, MNRAS, 432, 3401

Godfrey, L. E. H., \& Shabala, S. S. 2016, MNRAS, 456, 1172

Greenhill, L. J., Booth, R. S., Ellingsen, S. P., et al. 2003, ApJ, 590, 162

Gueth, F., \& Guilloteau, S. 1999, A\&A, 343, 571

Gueth, F., Guilloteau, S., \& Bachiller, R. 1996, A\&A, 307, 891

Heisler, C. A., \& Vader, J. P. 1994, AJ, 107, 35

Helou, G., Soifer, B. T., \& Rowan-Robinson, M. 1985, ApJ, 298, L7

Hickox, R. C., Mullaney, J. R., Alexander, D. M., et al. 2014, ApJ, 782, 9

Hujeirat, A., Livio, M., Camenzind, M., \& Burkert, A. 2003, A\&A, 408, 415

Imanishi, M. 2006, AJ, 131, 2406

Imanishi, M., Nakanishi, K., Tamura, Y., \& Peng, C. 2009, AJ, 137, 3581

Ishibashi, W., \& Fabian, A. C. 2015, MNRAS, 451, 93

King, A. R., \& Pringle, J. E. 2007, MNRAS, 377, L25

Konigl, A., \& Pudritz, R. E. 2000, Protostars and Planets IV, 759

Kormendy, J., \& Ho, L. C. 2013, ARA\&A, 51, 511

Kristensen, L. E. 2015, IAU General Assembly, Meeting 29, 2257263

Kristensen, L. E., Klaassen, P. D., Mottram, J. C., Schmalzl, M., \& Hogerheijde, M. R. 2013, A\&A, 549, L6

Krolik, J. H., \& Hawley, J. F. 2015, ApJ, 806, 141

Kwon, W., Fernández-López, M., Stephens, I. W., \& Looney, L. W. 2015, ApJ 814,43

Larson, R. B. 2010, Rep. Prog. Phys., 73, 014901

Lu, J.-F., \& Zhou, B.-Y. 2005, ApJ, 635, L17

Luque-Escamilla, P. L., Martí, J., \& Martínez-Aroza, J. 2015, A\&A, 584, A122

Martí-Vidal, I., Marcaide, J. M., Alberdi, A., et al. 2011, A\&A, 533, A111

Masciadri, E., \& Raga, A. C. 2002, ApJ, 568, 733

Matsushita, S., Muller, S., \& Lim, J. 2007, A\&A, 468, L49

McAlpine, K., Prandoni, I., Jarvis, M., et al. 2015, PoS(AASKA14)083

Milosavljević, M., \& Merritt, D. 2003, ApJ, 596, 860

Morganti, R., Oosterloo, T., Oonk, J. B. R., Frieswijk, W., \& Tadhunter, C. 2015, A\&A, 580, A1

Nakai, N., Hayashi, M., Handa, T., et al. 1987, PASJ, 39, 685

Nayakshin, S. 2014, MNRAS, 437, 2404

Nayakshin, S., Power, C., \& King, A. R. 2012, ApJ, 753, 15

Nixon, C., \& King, A. 2013, ApJ, 765, L7

Pyrzas, S., Steenbrugge, K. C., \& Blundell, K. M. 2015, A\&A, 574, A30

Raga, A., \& Cabrit, S. 1993, A\&A, 278, 267

Raga, A., Cabrit, S., Dougados, C., \& Lavalley, C. 2001, A\&A, 367, 959

Rosen, A., \& Smith, M. D. 2004, MNRAS, 347, 1097

Roussel, H., Helou, G., Beck, R., et al. 2003, ApJ, 593, 733

Roussel, H., Helou, G., Smith, J. D., et al. 2006, ApJ, 646, 841

Sakamoto, K., Aalto, S., Costagliola, F., et al. 2013, ApJ, 764, 42

Sakamoto, K., Aalto, S., Combes, F., Evans, A., \& Peck, A. 2014, ApJ, 797, 90

Santangelo, G., Codella, C., Cabrit, S., et al. 2015, A\&A, 584, A126
Santiago-García, J., Tafalla, M., Johnstone, D., \& Bachiller, R. 2009, A\&A, 495, 169

Sbarrato, T., Padovani, P., \& Ghisellini, G. 2014, MNRAS, 445, 81

Sikora, M., Stasińska, G., Kozieł-Wierzbowska, D., Madejski, G. M., \& Asari, N. V. 2013, ApJ, 765, 62

Spoon, H. W. W., Marshall, J. A., Houck, J. R., et al. 2007, ApJ, 654, L49

Steffen, W. 1997, Vistas Astron., 41, 71

Storchi-Bergmann, T., Lopes, R. D. S., McGregor, P. J., et al. 2010, MNRAS, 402, 819

Sturm, E., González-Alfonso, E., Veilleux, S., et al. 2011, ApJ, 733, L16

Veilleux, S., Tully, R. B., \& Bland-Hawthorn, J. 1993, AJ, 105, 1318

Wada, K. 2015, ApJ, 812, 82

Wagner, A. Y., \& Bicknell, G. V. 2011, ApJ, 728, 29

Walter, F., Weiss, A., \& Scoville, N. 2002, ApJ, 580, L21

Wu, P.-F., Takakuwa, S., \& Lim, J. 2009, ApJ, 698, 184

Xu, C., Baum, S. A., O’Dea, C. P., Wrobel, J. M., \& Condon, J. J. 2000, AJ, 120, 2950

1 Department of Earth and Space Sciences, Chalmers University of Technology, Onsala Observatory, 43992 Onsala, Sweden e-mail: saalto@chalmers.se

2 Institute of Astronomy and Astrophysics, Academia Sinica, PO Box 23-141, 10617 Taipei, Taiwan

3 Department of Astronomy, University of Wisconsin-Madison, 5534 Sterling, 475 North Charter Street, Madison WI 53706, USA

4 Department of Astrophysics, Astronomy \& Mechanics, Faculty of Physics, University of Athens, 15784 Panepistimiopolis Zografos, Greece

5 Kagoshima University, 890-0065 Kagoshima, Japan

6 Observatoire de Paris, LERMA (CNRS: UMR 8112), 61 Av. de l'Observatoire, 75014 Paris, France

7 Observatorio Astronómico Nacional (OAN) - Observatorio de Madrid, Alfonso XII 3, 28014 Madrid, Spain

8 Harvard-Smithsonian Center for Astrophysics, 60 Garden Street, Cambridge, MA 02138, USA

9 European Southern Observatory, Alonso de Córdova 3107, Vitacura, Santiago, Chile

10 Joint ALMA Observatory, Alonso de Córdova 3107, Vitacura, Santiago, Chile

11 Institut de Radio Astronomie Millimétrique (IRAM), 300 rue de la Piscine, Domaine Universitaire de Grenoble, 38406 St. Martin $\mathrm{d}^{\prime}$ Hères, France

12 Leiden Observatory, Leiden University, 2300 RA, Leiden, The Netherlands

13 University of Virginia, Charlottesville, VA 22904, USA, NRAO, 520 Edgemont Road, Charlottesville, VA 22903, USA

14 Finnish Centre for Astronomy with ESO (FINCA), University of Turku, Väisäläntie 20, 21500 Kaarina, Finland 


\section{Appendix A: Spectra}
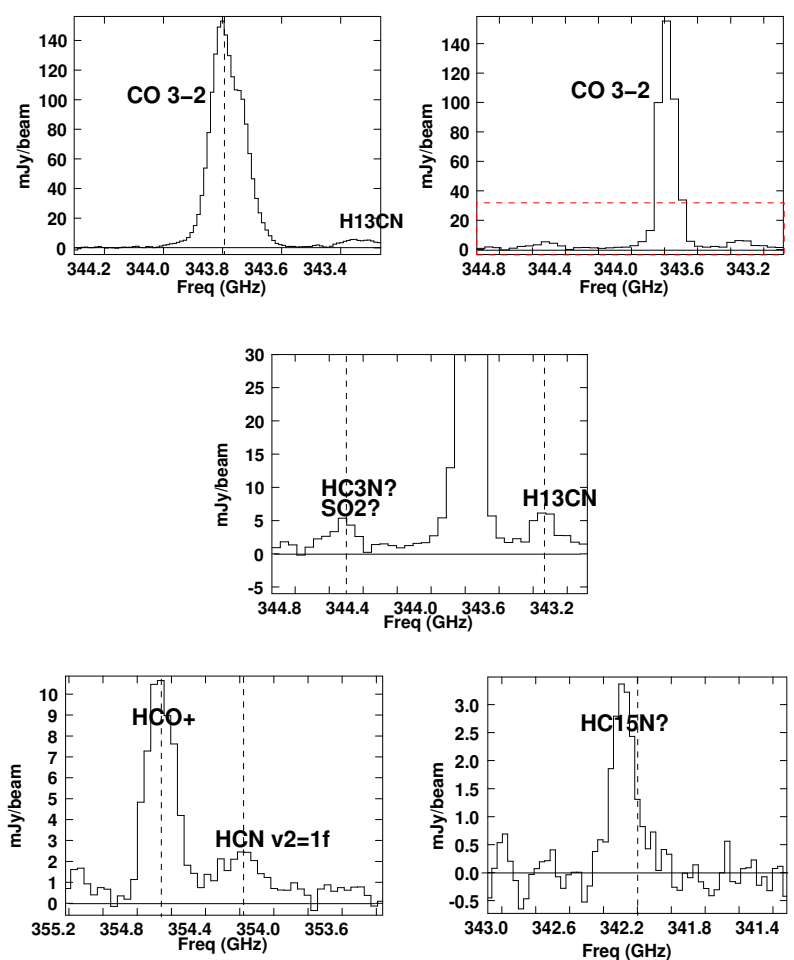

Fig. A.1. Spectra of the nuclear emission in NGC 1377. Dashed vertical line indicates $v=1740 \mathrm{~km} \mathrm{~s}^{-1}$. Top panels: CO 3-2 in high- (left) and low- (right) resolution spectral mode. The dashed red box of the right panel indicates the zoomed-in region in the next panel. Centre: zoomedin spectrum showing detections of $\mathrm{H}^{13} \mathrm{CN} J=4-3$ and either $\mathrm{HC}_{3} \mathrm{~N}$ $J=38-37 v_{4}=1, v_{7}=1$ or $\mathrm{SO}_{2} 16(4,12)-16(3,13)$. Bottom: panels showing detections of $\mathrm{HCO}^{+} J=4-3, \mathrm{HCN} J=4-3 v_{2}=1 f$ (left) and a line that we tentatively identify as $\mathrm{HC}^{15} \mathrm{~N} J=4-3$. All spectra apart from in panels 2 and 3 have Gaussian smoothed with FWHM of two channels. In panels 2 and 3 there has been no smoothing but frequency resolution is reduced by a factor of 3 (these data stem from another spectral window than that presented in the first panel).

In Fig. A.1 we present spectra towards the nucleus of NGC 1377. Apart from $\mathrm{CO} 3-2$ we detect $\mathrm{HCO}^{+}, \mathrm{H}^{13} \mathrm{CN} J=4-3$, vibrationally excited $\mathrm{HCN} J=4-3 v_{2}=1 f\left(T=E_{1} / k=1050 \mathrm{~K}\right)$. We detect a line at $v=345.5 \mathrm{GHz}$ which is either vibrationally excited $\mathrm{HC}_{3} \mathrm{~N} J=38-37 v_{4}=1, v_{7}=1 f\left(T=E_{1} / k=1891 \mathrm{~K}\right)$ or it is $\mathrm{SO}_{2} 6(4,12)-16(3,13)\left(T=E_{1} / k=148 \mathrm{~K}\right)$. In addition we detect a line at redshifted frequency $v=342.26 \mathrm{GHz}$ which we tentatively identify as $\mathrm{HC}^{15} \mathrm{~N} J=4-3$. In this case, the line would peak at $v=1670 \mathrm{~km} \mathrm{~s}^{-1}$ and thus be blueshifted with respect to the other lines by $60 \mathrm{~km} \mathrm{~s}^{-1}$. This type of shift could be caused by excitation, optical depth and/or abundance gradients and should be investigated in further studies since it may hold another clue to the nature of the nuclear emission of NGC 1377.

\section{Appendix B: Other potential explanations to the high velocity $\mathrm{CO} 3-2$ emission}

An orbiting object and/or two jets? Velocity variations in a PV diagram may also be caused by a jet launched from an orbiting object. In this case the velocity reversals can be dominated by the orbital motion in a near edge-on plane of rotation. A possibility would be a jet launched from one of two orbiting SMBHs. Masciadri \& Raga (2002) have discussed the similarities and differences between orbiting and precessing jets. However, without jet precession the velocity pattern will not fit the structure we see in the observed PV diagram - unless the jet symmetry axis is misaligned with respect to the axis of the plane of rotation. Both SMBHs could have jets and a combination of orientation and length of the jets could be put together to reproduce the observed PV diagram. However, this seems unlikely compared to the relatively simple scenario of one single precessing jet.

Jet shocks? A pulsed jet will have a sawtooth like pattern in its PV diagram along the jet major axis (e.g. Santiago-García et al. 2009). This pattern is caused by axial compression and lateral ejection of material inside the internal working surface. Santiago-García et al. (2009) point out that the effects are localized within the jet so it is not obvious how it would give rise to the large scale shifts and gradients seen here. However, internal and external shocks would be important for the jet of NGC 1377 and thus influence its velocity structure.

A bicone projection? Is it possible that the velocity reversals we observe in the PV diagram A (Fig. 3) is a projection effect, instead of the emission from a collimated jet? A tilted wideangle biconical outflow may result in projected, foreshortened blueshifted emission from the lower end of the cone, and redshifted (more elongated) emission from the back side of the cone (and vice versa on the underside of the cone). PV diagrams of these scenarios are, for example, presented by (e.g. Cabrit $\&$ Bertout 1986; Das et al. 2005; and Storchi-Bergmann et al. 2010).

In Fig. B.1 we show a sketch of a cone (displayed from two angles) with the northern part tilted towards us. Schematic PV diagrams along and transverse to the projected cone symmetry axis are shown in Fig. B.2. (Note that it is a very simple cone model with uniform density. The PV diagram would be much more complicated for a non-uniform cone, or multiple cones.) The resulting PV diagram along the main axis has two scenarios: one with constant outflow velocity, and one where the gas is first accelerating and then decelerating. It is likely possible to find an outflow velocity scenario that can at least produce a reasonable fit to the PV along the jet axis (A in Fig. 3), if the northern cone is tilted towards us and the opening angle is large, $>45^{\circ}$. This orientation of a wide angle cone is, however, inconsistent with the optical dust structure found by Roussel et al. (2006, their Fig. 1) and Heisler \& Vader (1994, their Fig.4c), which would require the northern cone to be tilted away from us.

Another important argument against the cone-projection model is the shape of the observed PV diagrams transverse to the jet symmetry axis (panels $\mathrm{C}-\mathrm{F}$ in Fig. 3). For a cone, the PV diagrams perpendicular to the major axis will always be ellipses (see schematic PV diagrams in Fig. B.2). And, when the top cone is tilted towards us, there should be a broad (in space) blueshifted emission component to the north. The observed PV diagrams transverse to the jet axis (Fig. 3) do, however, not show this structure. In Fig. B.3 we show the PV diagrams D and E from Fig. 3 with the expected PV diagram of a tilted cone indicated by dashed lines. Instead of tracing out the curved front ellipse, the maximum velocity is structured in a spatially unresolved tounge-like shape of broad emission.

We note that this exercise is not an attempt to model the minor-axis structure of the low-velocity gas as discussed in Sect. 4.2. The low-velocity gas may (at least partially) originate in a cone-like slow outflow, which we suggest is interacting with the molecular jet. An attempt to link it to the optical dust structure mentioned above would require its southern part to be at least slightly directed towards us. 
Edge-on view

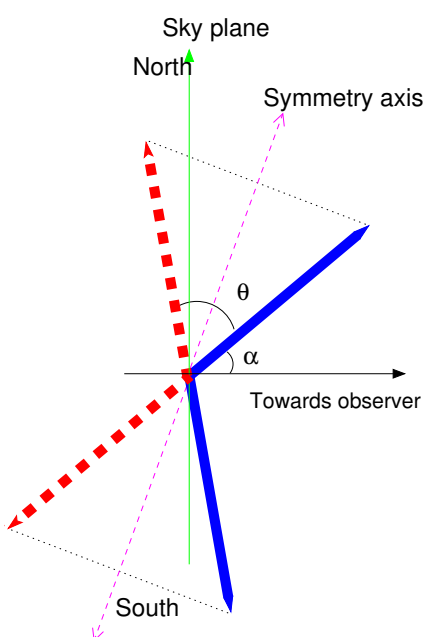

Face-on view

North

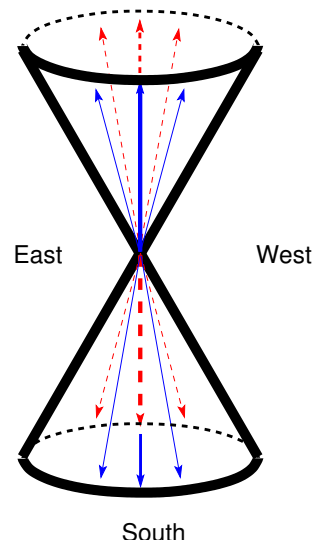

Fig. B.1. Sketch of a hollow cone with opening angle $60^{\circ}$ and tilt angle $40^{\circ}$ towards the observer.
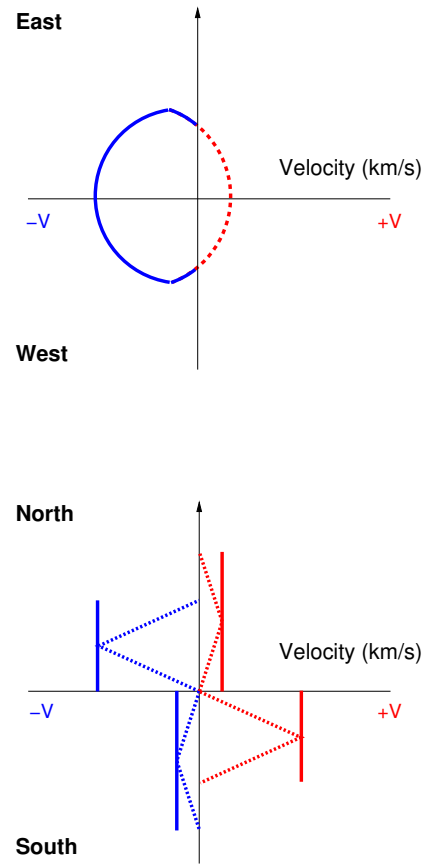

Fig. B.2. Schematic PV diagrams of a cone with its top (northern) part tilted towards us. Top panel: cut perpendicular to the cone axis showing the elliptical PV diagram through the cone. Bottom panel: cut along the cone major axis. We show two simplified cases: the straight solid lines show the PV diagram of outflowing gas along the cone walls of constant velocity. The dashed lines show the generic PV diagram along the axis of a cone where the gas is first linearly accelerating and then decelerating to zero velocity.
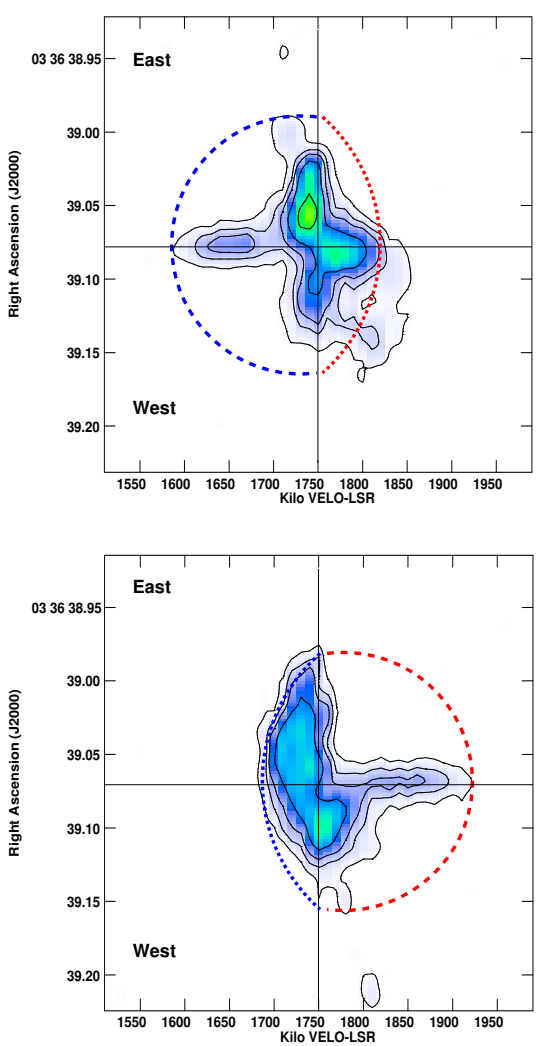

Fig. B.3. PV diagram, showing gas velocities in a slit across the jet axis at \pm 0 ' 3 . Contour levels are $3.1 \times(1,2,4,8,16,32) \mathrm{mJy} \mathrm{beam}^{-1}$. The colour scale ranges from -11 to $156 \mathrm{mJy}^{-1}$ beam $^{-1}$. The dashed semiellipticals show the PV diagram expected from a projected wide-angle cone It is clear that this model does not fit the data. 